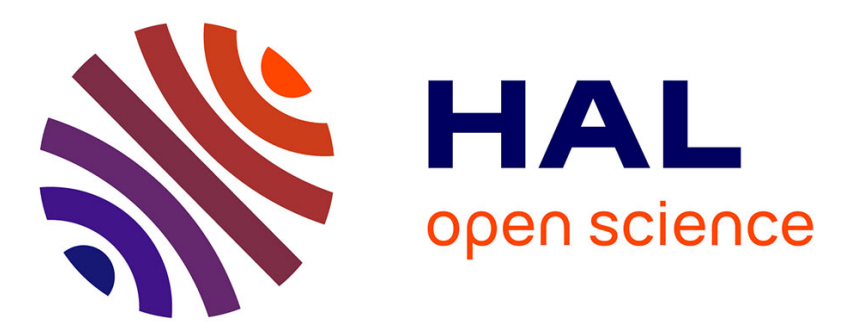

\title{
Combined effects of bed friction and emergent cylinder drag in open channel flow
}

\author{
V. Dupuis, Sébastien Proust, Céline Berni, André Paquier
}

\section{To cite this version:}

V. Dupuis, Sébastien Proust, Céline Berni, André Paquier. Combined effects of bed friction and emergent cylinder drag in open channel flow. Environmental Fluid Mechanics, 2016, 16 (6), pp.11731193. 10.1007/s10652-016-9471-2 . hal-01709769

\section{HAL Id: hal-01709769 \\ https://hal.science/hal-01709769}

Submitted on 15 Feb 2018

HAL is a multi-disciplinary open access archive for the deposit and dissemination of scientific research documents, whether they are published or not. The documents may come from teaching and research institutions in France or abroad, or from public or private research centers.
L'archive ouverte pluridisciplinaire HAL, est destinée au dépôt et à la diffusion de documents scientifiques de niveau recherche, publiés ou non, émanant des établissements d'enseignement et de recherche français ou étrangers, des laboratoires publics ou privés. 


\title{
Combined effects of bed friction and emergent cylinder drag in open channel flow
}

\author{
Victor Dupuis · Sébastien Proust · Céline Berni · \\ André Paquier
}

Received: date / Accepted: date

\begin{abstract}
Open channel flows subjected to a longitudinal transition in roughness, from bed friction to emergent cylinder drag and vice versa, are investigated experimentally in an 18 $\mathrm{m}$ long laboratory flume. These are compared to uniform flows subject to (1) bed roughness only and (2) an array of emergent vertical cylinders installed on bed roughness. The nearbed region is investigated in detail for uniform flows through the cylinder array. The water column can be divided into two parts: a region of constant velocity and a boundary layer near the channel bed. In the latter region, a local increase in velocity, or velocity bulge, is observed in line of a cylinder row. The velocity bulge may be related to the disorganization of the von Kármán vortex street by the bed-induced turbulence, resulting in reduced momentum loss in the cylinder wake. The boundary layer height is found to be independent of water depth and bed roughness (smooth or rough bottom). Strong oscillations of the free surface (seiching) are observed. Oscillation amplitude is dependent on the longitudinal position within the cylinder array and is found to decrease with decreasing array length. When water depth/boundary layer height ratio is close to unity, the disorganization of the von Kármán vortex street throughout the water column prevents seiching from occurring. In the case of roughness transition flows, the water depth is found to vary only upstream of the change in roughness. Vertical profiles of velocity and turbulence are self-similar upstream of the transition and collapse with the uniform flow profiles. Downstream of the roughness change, velocity and turbulence vary over a distance of 35 to 50 times the water depth. Roughness transition flows show that seiching is lowered by flow non-uniformity. A 1D momentum equation integrating bed friction and drag force exerted by the cylinder array predicts accurately the water surface profile $(0.9 \%$ mean relative error). The computed profiles show that, upstream of the transition, flow depth varies over a distance of about 2600 times the uniform water depth of the upstream roughness. The $1 \mathrm{D}$ equation is solved analytically for zero bed friction.
\end{abstract}

V. Dupuis $\cdot$ S. Proust $\cdot$ C. Berni $\cdot$ A. Paquier

Irstea, UR HHLY Hydrologie-Hydraulique, Centre de Lyon-Villeurbanne

5 rue de la Doua BP32108

F-69616 Villeurbanne Cedex, France

Tel.: +33-47-2208787

Fax: +33-47-8477875

E-mail: victor.dupuis@irstea.fr·sebastien.proust@irstea.fr·celine.berni@irstea.fr·andre.paquier@irstea.fr 
Keywords laboratory study $\cdot$ roughness transition $\cdot$ rigid vegetation $\cdot$ free surface oscillation $\cdot$ seiching

\section{Introduction}

Longitudinal transitions in hydraulic roughness often characterise environmental open channel flows, for example, where floodplain vegetation changes along a river. A first common form of transition is a sudden change in bed roughness. This has been experimentally investigated by Robert et al. [22], Chen and Chiew [4], Carravetta and Della Morte [2]. There is a second type of roughness transition between bed roughness and emergent elements, for example a longitudinal transition in a floodplain between a deeply flooded meadow and a wood. This laboratory study focuses on the latter type of transition featuring a meadow modelled by plastic grass and trees represented by an array of emergent vertical cylinders.

Longitudinal transitions between two bed roughnesses have been widely investigated in the literature for air flows in wind tunnels $[1,18,6]$ and in air ducts [25]. The sudden or so-called step change in roughness generates a new layer, which starts at the wall and grows vertically towards the free stream region (or towards the duct axis).

Longitudinal changes in bed roughness for open channel flows have been investigated much less. Chen and Chiew [4] have shown that velocity profile adjustment to the downstream roughness does not extend gradually from the wall to the free stream region, as it does in air flows, but simultaneously over the entire flow depth. Chen and Chiew [5] and Carravetta and Della Morte [2] have indicated that additional effects can appear, such as shear stress overshoot, if the roughness step change is associated with a step change in bed level.

Flows through emergent elements, usually vertical cylinder arrays, have been mainly studied under uniform flow conditions $[19,14,15]$. Flow through an emergent cylinder array is driven by a volume drag force, which generates turbulence throughout the water column. This results in constant mean velocity and turbulence quantities throughout the depth. The physical processes are thus very different from those resulting from a boundary layer on a bed roughness, for which turbulence production is limited to the near-bed region [21]. Flows through emergent cylinder arrays are also associated with free surface oscillations, namely transverse waves whose amplitude can reach $35 \%$ of the water depth [30].

Zong and Nepf [31] and Rominger and Nepf [23] have investigated flows through patches of cylinder arrays occupying only a part of the channel width. Such flows are characterised by a lateral mixing layer developing between the flow through the cylinder array and the free stream. To the author's knowledge, a step change from bed friction to emergent element drag across the entire channel width has not been addressed in the literature. In the present study, we investigate experimentally the transition from (1) bed roughness, typically a fully submerged dense meadow in a river floodplain, to (2) an array of emergent cylinders, typically emergent trees in a wooded floodplain, and vice versa. The cylinder array was installed on the bed roughness to more closely represent actual field conditions.

The first objective of this study is to assess the combined effects of bed friction and cylinder drag within the cylinder array. The second objective is to assess the response of flow depth and velocity to longitudinal change from bed friction to emergent cylinder drag. In particular, the distances upstream and downstream of the transition, beyond which the flow can be considered as uniform, need to be evaluated.

The experimental setup is described in Section 2 and includes five flow configurations. Each type of roughness (bed roughness and cylinder array) is investigated under uniform 
flow conditions. Special attention is given to the flow in the near-bed region within the cylinder array and to the free surface oscillation phenomenon (seiching). The results of this part of the study are presented in Section 3. The uniform flows are used as references for studying the roughness transitions, which are presented in Section 4. The water depth response to the roughness transition is first investigated and a 1D momentum equation is used to predict the water surface profile. The response of velocity and turbulence is then analysed. Finally, Section 4 ends with the effect of flow non-uniformity on free surface oscillations.

\section{Experimental setup and methodology}

Experiments were performed in an $18 \mathrm{~m}$ long, $1 \mathrm{~m}$ wide glass-wall flume in the Hydraulics and Hydromorphology Laboratory at Irstea Lyon-Villeurbanne, France. The longitudinal bottom slope was $S_{0}=1.05 \mathrm{~mm} / \mathrm{m}$. Longitudinal, lateral and vertical coordinates were denoted by $x, y$ and $z$ respectively. The channel cross section was rectangular and its width denoted by $B=1 \mathrm{~m}$.

Four main flow configurations were investigated as outlined in Fig. 1a: uniform flows over meadow-type vegetation (M) and through wood-type vegetation $(\mathrm{W})$, transitions from meadow to wood (MW) and from wood to meadow (WM). An additional uniform flow with the wood-type vegetation over a smooth glass bed (W0) was also studied. Table 1 reports the 13 flow cases experimented. The notations in the first column of Table 1 refer to the different flow configurations. The roughness upstream or downstream of the roughness step change is called the upstream or the downstream roughness respectively.

For the uniform flows, three flow rates were considered for each roughness type. For meadow-type vegetation, the three flow cases correspond to different degrees of blade submergence. For wood-type vegetation, the three flow cases correspond to different bed friction/cylinder drag ratios. In each case, the roughness was uniformly distributed along the whole flume $(18 \mathrm{~m})$. The downstream boundary condition (weir level) was adjusted to obtain a constant water depth along the flume (within $\pm 1 \mathrm{~mm}$ ). Flow rates and weir levels for the roughness transition experiments corresponded to those of uniform flows over the downstream roughness. The roughness step change was located at mid-length of the flume, i.e. $9 \mathrm{~m}$ downstream of the inlet tank. This location is also the origin of the longitudinal axis $(x=0)$.

Plastic artificial grass was used to model meadow-type vegetation. The blades of grass were $5 \mathrm{~mm}$ long and very dense such that the flow velocity and flow rate within the canopy could be assumed to be negligible. The blades of grass were rigid and of uniform length, so the vertical axis origin $(z=0)$ can be accurately defined as the tips of the blades. Wood-type vegetation was modelled using circular wood cylinders uniformly distributed in staggered rows (see Fig. 1b). The cylinder diameter was $D=10 \mathrm{~mm}$ and the cylinder density was $N=81$ cylinders. $\mathrm{m}^{-2}$. On a 1:100 scale, these cylinders modelled $1 \mathrm{~m}$ diameter trees separated by a mean distance of $11.3 \mathrm{~m}$. According to Terrier [27], this corresponds to a typical riparian forest in the lower reaches of the River Rhône (France).

The cylinders were installed on the plastic grass (except for test case W0-Q15 for which the bed was smooth glass) and were held in place from above by a wooden superstructure (see Fig. 2a). Cylinder lateral rows were termed even or odd depending on their even or odd numbers of cylinders. The array was composed of an elementary $80 \mathrm{~mm} \times 80 \mathrm{~mm}$ pattern as shown in Fig. 1b. Points A, B and C are velocity measuring positions. Point A is $4 \mathrm{~cm}$ 
(a)

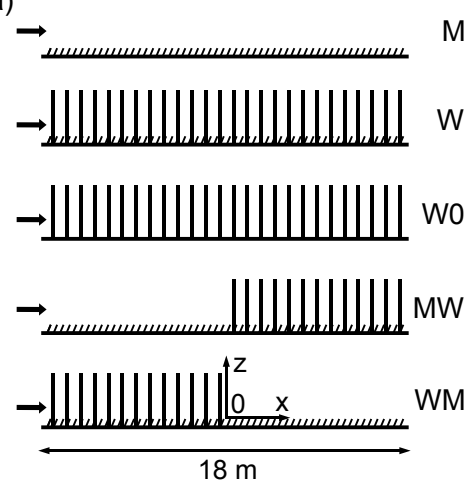

(b)

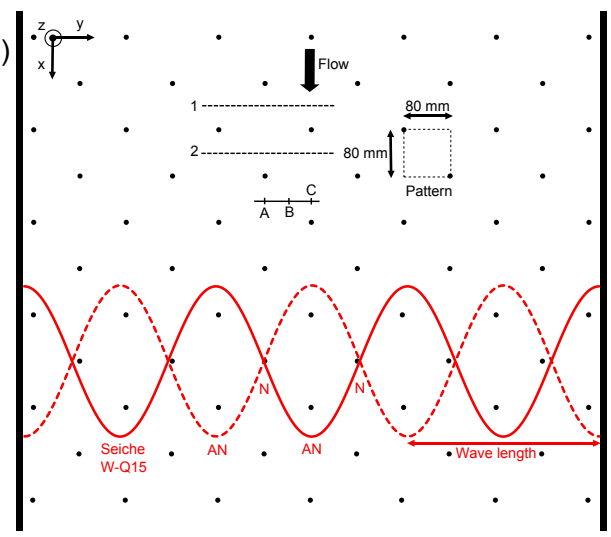

Fig. 1 (a) Side view of the flow configurations. (b) Plan view of cylinder array. Points A, B and C are velocity measuring positions. Profiles 1 and 2 are measuring positions of water depth for seiching. Red indications relate to seiching in test case W-Q15. Points AN and N are antinodes and nodes respectively.

Table 1 Test case flow conditions: flow rate $Q$, water depth $H$, bulk velocity $U_{Q}=Q /(B H)$, Froude number $F r=U_{Q} / \sqrt{g H}$, Reynolds number $R e=4 U H / v$, ratio $R$ between bed friction and drag force (see Eq. 5). Variation range between the most upstream measuring point $x=-8 \mathrm{~m}$ and mean value in downstream reach are given for roughness transition flows. $R$-ratio is only given for the wooded area (undefined for area outside cylinder array) for roughness transition flows.

\begin{tabular}{cccccccc}
\hline Test case & Flow configuration & $\begin{array}{c}Q \\
\left(\mathrm{~L} . \mathrm{s}^{-1}\right)\end{array}$ & $\begin{array}{c}H \\
(\mathrm{~mm})\end{array}$ & $\begin{array}{c}U_{Q} \\
\left(\mathrm{~cm}^{-1}\right)\end{array}$ & $F r$ & $\begin{array}{c}R e \\
\left(10^{-3}\right)\end{array}$ & $R$ \\
\hline M-Q7 & Uniform meadow & 7 & 35 & 20.6 & 0.35 & 29 & \\
M-Q15 & Uniform meadow & 15 & 55 & 27.3 & 0.37 & 60 & \\
M-Q50 & Uniform meadow & 50 & 116.5 & 42.9 & 0.40 & 200 & 0.25 \\
W-Q7 & Uniform wood & 7 & 55 & 12.7 & 0.17 & 28 & 0.10 \\
W-Q15 & Uniform wood & 15 & 113 & 13.3 & 0.13 & 60 & 0.06 \\
W-Q21 & Uniform wood & 21 & 152 & 13.8 & 0.11 & 84 & 0.04 \\
W0-Q15 & Uniform wood with smooth bed & 15 & 109 & 13.8 & 0.13 & 60 & 0.25 \\
MW-Q7 & Transition from meadow to wood & 7 & $49-56$ & $14.3-12.5$ & $0.21-0.17$ & $28-28$ & 0.10 \\
MW-Q15 & Transition from meadow to wood & 15 & $104-113$ & $14.4-13.3$ & $0.14-0.13$ & $60-60$ & 0.06 \\
MW-Q21 & Transition from meadow to wood & 21 & $143-152$ & $14.7-13.8$ & $0.12-0.11$ & $84-84$ & $0.31-0.46$ \\
WM-Q7 & Transition from wood to meadow & 7 & $47-35$ & $14.9-20.0$ & $0.22-0.34$ & $28-28$ & $0.00-16-0.25$ \\
WM-Q15 & Transition from wood to meadow & 15 & $76-56$ & $19.7-26.8$ & $0.23-0.36$ & $60-60$ & 0.16 \\
WM-Q50 & Transition from wood to meadow & 50 & $168-119$ & $29.4-42.7$ & $0.23-0.40$ & $200-200$ & $0.06-0.09$ \\
\hline
\end{tabular}

downstream of a cylinder, Point $\mathrm{C}$ is $4 \mathrm{~cm}$ upstream of a cylinder and Point $\mathrm{B}$ is in the free stream between two longitudinal rows of cylinders.

Water depths were measured using an electronic stage gauge (Mitutoyo 570-302) with an accuracy of $\pm 0.5 \mathrm{~mm}$ and free surface oscillations (seiching) were investigated with an ultrasonic sensor (Baumer UNDK20I69) with a repeatability lower than $0.5 \mathrm{~mm}$. Mean velocity and turbulence fluctuations were recorded using a side-looking ADV probe (Nortek Vectrino Plus). The recording time at each point was $180 \mathrm{~s}$ and the acquisition rate was $100 \mathrm{~Hz}$. This was found to be sufficient for obtaining converged first and second statistical moments. The ADV raw data were filtered using WinADV freeware based on the de-spiking concept developed by Goring and Nikora [9]. The tracers used for the ADV measurements were hydrogen micro-bubbles, generated by an iron anode (wire) positioned on the flume bottom approximately $3 \mathrm{~m}$ upstream of the measuring point. Preliminary measurements showed that this device did not disturb the flow at the measuring location. In addition, the 
(a)

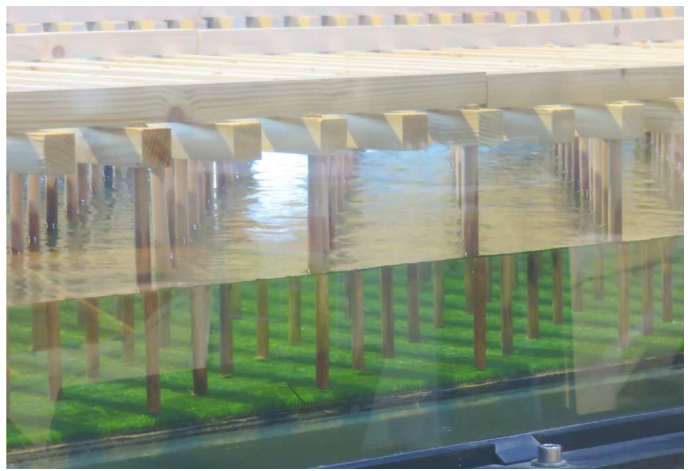

(b)

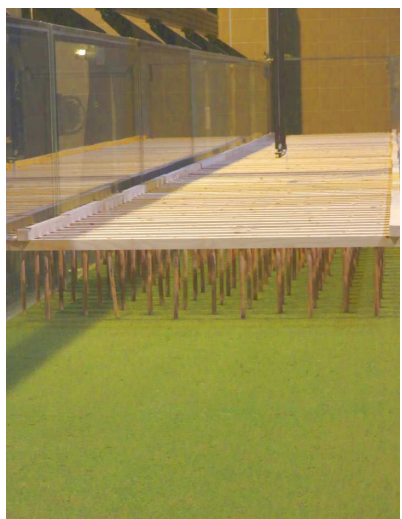

Fig. 2 (a) Side view of cylinder array over bed roughness. (b) Flume configuration for meadow-to-wood transition (viewed from upstream).

micro-bubble buoyancy was negligible (rising velocity about $5 \cdot 10^{-6} \mathrm{~m} \cdot \mathrm{s}^{-1}$ ). ADV sensor accuracy was $0.5 \%$ of the measured mean velocity (according to the manufacturer). Some uniform flow velocity profiles were measured using a LaVision 2D PIV laser system. The laser sheet entered the flow from the flume bottom through a narrow slot cut in the plastic grass and illuminated a vertical plane $(x, z)$. ADV probe measurements were used to check that the slot did not influence the local flow field. Each PIV profile presented is a time-based average profile for 150 images taken at $5 \mathrm{~Hz}$ and a space-based average over a $100 \mathrm{~mm}$ longitudinal distance for flows over bed roughness and over a $30 \mathrm{~mm}$ longitudinal distance for flows through the cylinder array. The PIV measuring location is at Point $\mathrm{C}$ for flows through the cylinder array (see Fig. 1b), i.e. $40 \mathrm{~mm}$ upstream of a cylinder centre (space averaging between 25 and $65 \mathrm{~mm}$ upstream of cylinder centre). Unless otherwise stated, the ADV probe was used for measuring all velocities and turbulence quantities presented herein. The flow rate was automatically regulated by control valves and measured by an electromagnetic flowmeter.

\section{Uniform flows}

This section focuses on uniform flows subjected to (1) bed friction alone (case M in Fig. 1a) and (2) combined emergent cylinder drag and bed friction (cases W and W0 in Fig. 1a). Flow is considered uniform when the flow parameters (water depth, mean velocity, turbulence) remain constant in the downstream direction. In the case of flow through an emergent cylinder array, uniformity is reached when the flow no longer varies from one elementary pattern to another.

\subsection{Bed friction}

The Manning roughness coefficient of the meadow was evaluated as $n=0.0166 \mathrm{~s} \cdot \mathrm{m}^{-1 / 3}$ based on uniform test cases M-Q7, M-Q15 and M-Q50. Variation in this $n$-value for the three M-cases was lower than $\pm 2.5 \%$. It should be noted that bed and side walls do not have the same roughness. The heterogeneous roughness along the wetted perimeter can 


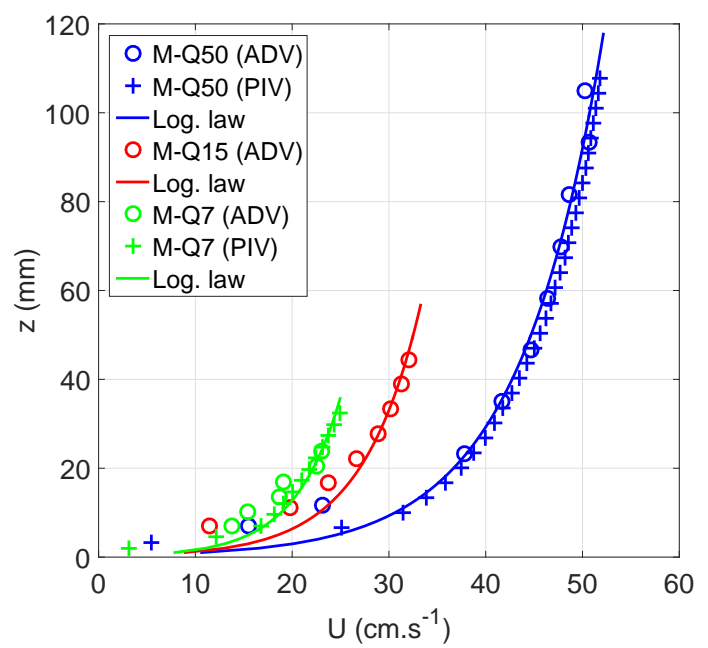

Fig. 3 Vertical profiles of mean longitudinal velocity for uniform flows over a meadow-type roughness for flow rates $Q=7,15$ and 50 L.s ${ }^{-1}$.

be approximately taken into account using a Manning formula for composite roughness [28]. The Manning coefficient of the glass wall was estimated in previous experiments: $n_{\text {glass }}=0.0096 \mathrm{s.m}-1 / 3$ with variations lower than $4 \%$ in the range of Reynolds number values considered.

Figure 3 shows the vertical profiles of mean longitudinal velocity $U$ for the above three M-cases. Open circles are used for ADV probe measurements and crosses for PIV measurements, which were only available for test cases M-Q7 and M-Q50. ADV and PIV measurements collapse in the upper water column for $z>20 \mathrm{~mm}$. Below this level, the ADV probe underestimates the mean velocity. In this region, the ADV measurements are scattered and the signal quality is lower; this was not observed with a smooth glass bed. The plastic grass therefore appears to cause defective behaviour of the ADV in this region: it may induce acoustic signal interference or ejection of the tracer from the near-bed region. Measurement difficulties with ADV probes in near-wall regions have been regularly reported [3]. The accuracy of the PIV measurements in the near-bed region has been verified by comparing them with a logarithmic law as shown in Fig. 3.

\subsection{Cylinder drag combined with bed friction}

This section considers uniform flows through an array of rigid vertical emergent cylinders (cases $\mathrm{W}$ and W0). It is important to note that, compared with previous studies of cylinder arrays $[19,14,15]$, the characteristic length of the bed roughness is of the same order of magnitude as the cylinder diameter in this case: $k_{S} / D \approx 0.7$, where $k_{S}$ is the equivalent sand grain size of the bed roughness.

\subsubsection{Relative weights of bed friction and drag}

The flow resistance through a cylinder array is caused by the combination of cylinder drag force and bed friction. According to Nepf [16], the volume force $F_{\text {arr }}$ exerted by a cylinder 
array can be expressed as:

$$
F_{\text {arr }}=\frac{1}{2} \rho a C_{D} U_{Q}^{2}
$$

where $a$ is the frontal area per unit volume of cylinder array ( $a=N D$ for circular cylinders; $a=0.81 \mathrm{~m}^{-1}$ in the present case), $C_{D}$ is the drag coefficient of each cylinder and $U_{Q}$ is the bulk velocity. Neglecting the friction on the glass side walls, a 1D force balance between the driving gravity force, the bed friction force and the drag force leads to:

$$
\rho g H S_{0}=\tau_{b}+\frac{1}{2} \rho a C_{D} H U_{Q}^{2}
$$

where $S_{0}$ is the channel slope, $H$ is the water depth and $\tau_{b}$ is the bed shear stress. It should be noted that the fraction of the volume occupied by the cylinders $\left(\approx 6.10^{-3}\right)$ is neglected in this equation.

According to Kothyari et al. [13], the drag coefficient of one cylinder in a cylinder array is a function of the area concentration of cylinders $a D$ and of the cylinder Reynolds number $R e_{D}=U_{Q} D / v$. Using the empirical formula of Kothyari et al. [13] and comparing with the $C_{D}$-value recommended by Nepf [16], we take $C_{D}=1.2$ in the present case. Using the Manning roughness coefficient $n$ determined in Section 3.1 for the bed roughness, the bed shear stress is:

$$
\tau_{b}=\frac{\rho g n^{2} U_{Q}^{2}}{H^{1 / 3}} .
$$

Combining Eqs. 2 and 3 gives the stage-discharge relationship:

$$
Q=\left(\frac{H^{3} B^{2} g S_{0}}{g n^{2} H^{-1 / 3}+\frac{1}{2} a C_{D} H}\right)^{1 / 2} .
$$

Equation 4 is based on the assumption that the overall flow resistance is given by simply summing the two contributions, i.e. bed shear stress and drag. This is not the case in general: bed shear stress is actually affected by the cylinders (Eq. 3 is valid for bed roughness alone) and, conversely, bed friction affects the drag in the near-bed region, resulting in a modified mean drag coefficient. The uniform flow depths predicted by Eq. 4 match the experimental data $(2.7 \%$ mean relative error), indicating that the previous assumption is consistent in the present case.

The effect of bed friction relative to drag force can be quantified using the ratio between the two terms on the right-hand side of Eq. 2:

$$
R=\frac{2 g n^{2}}{a C_{D} H^{4 / 3}}
$$

The $R$-ratios for the three test cases are reported in Table 1 .

Drag forces act as a volume force on the fluid and therefore make the velocity field constant in the vertical direction, except in the near-bed region, where bed friction influences the flow and a boundary layer forms $[19,14,15]$. The water column can thus be divided into two parts: (1) a region of constant velocity in the upper part of the water column, termed here the constant-velocity region and (2) a boundary layer, defined as the region in which the velocity is variable. The space-averaged velocity in the constant-velocity region $\langle U\rangle_{\text {drag }}$ is obtained by assuming that the bed shear stress is zero in Eq. 2:

$$
\langle U\rangle_{d r a g}=\sqrt{\frac{2 g S_{0}}{a C_{D}}} .
$$


(a)

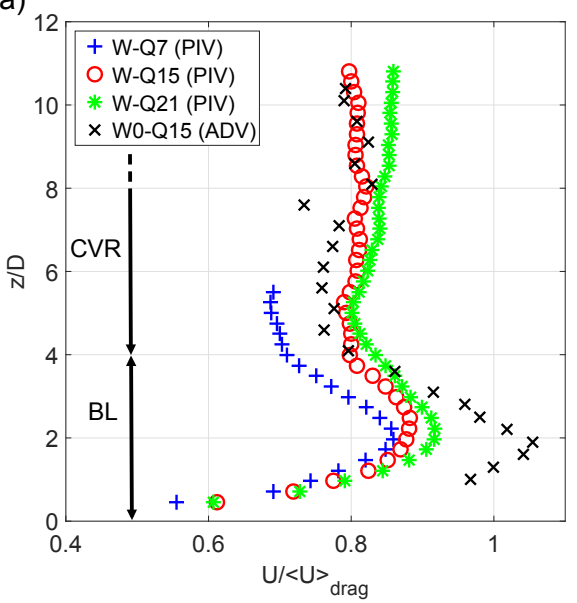

(b)

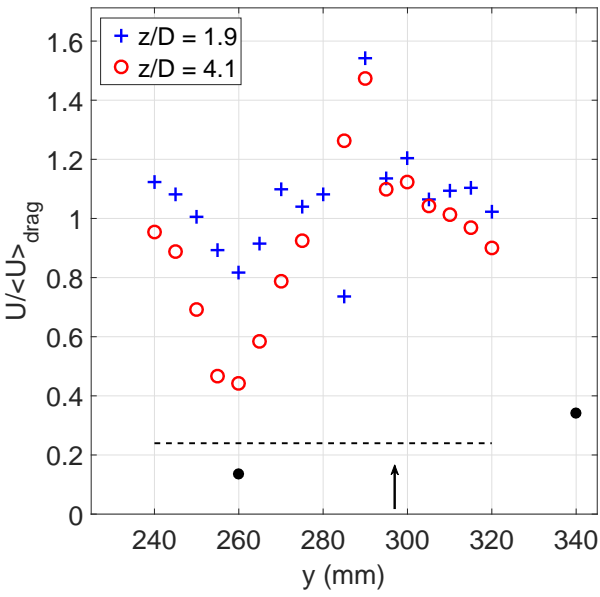

Fig. 4 (a) Vertical profiles of mean longitudinal velocity $U$ for uniform flows at Point C of the cylinder array, i.e. $40 \mathrm{~mm}$ upstream of the cylinder axis and at $y=340 \mathrm{~mm}$. BL is the boundary layer and CVR the constantvelocity region. (b) Lateral profiles of mean longitudinal velocity $U$ for W0-Q15 at two different heights: near the velocity bulge maximum $(z / D=1.9)$ and in the constant-velocity region $(z / D=4.1)$; black solid circles indicate cylinder positions (not to scale), the dashed line is the measuring position and the arrow shows the flow direction. The velocity is normalised by the spatially averaged velocity $\langle U\rangle_{\text {drag }}$ in the constant-velocity region (Eq. 6) and the vertical coordinate is normalised by the cylinder diameter.

The velocity $\langle U\rangle_{d r a g}$ is independent of water depth, according to the fact that the flow is driven by two volume forces only: gravity and drag. In the present case $\langle U\rangle_{\text {drag }}=$ $14.6 \mathrm{~cm} \cdot \mathrm{s}^{-1}$. The difference between this value and the bulk velocity (see Table 1) also quantifies the influence of the bed friction on the flow.

\subsubsection{Near-bed region}

Figure 4a shows vertical profiles of longitudinal mean velocity at Point C (see Fig. 1b), i.e. $40 \mathrm{~mm}$ upstream of a cylinder, for the three test cases with the rough bed (cases W, measurements using PIV) and for the test case with the smooth glass bed (case W0, measurements using ADV probe). The longitudinal velocity is normalised with the velocity $\langle U\rangle_{\text {drag }}$. For the highest flow rate (W-Q21), the velocity profile is cut and continues outside the PIV measuring window, but it can be assumed to be constant in the upper water column. Water column separation between the constant-velocity region (CVR) and the boundary layer (BL) is shown in Fig. 4. The boundary layer height $\delta$ is the same for the four cases $(\delta \approx 40 \mathrm{~mm})$, and appears to be independent of the water depth and the type of bed roughness. Length $\delta$ is therefore probably dependent on the geometry of the cylinder array only (cylinder density, cylinder diameter, cylinder arrangement). For test case W-Q7 with a relative high $R$-ratio, only a very small constant-velocity region can be distinguished and the velocity in this region is smaller than that in other cases. As Point $\mathrm{C}$ is aligned with a longitudinal row of cylinders, the local constant velocity is lower than the mean uniform velocity $\langle U\rangle_{\text {drag }}$ (spatially averaged over a pattern).

A local velocity increase is observed in the near-bed region. This velocity bulge reaches its maximum at $z / D=2(z=20 \mathrm{~mm})$; its position is independent of water depth and of bed 
roughness (rough or smooth). The intensity of the velocity bulge is higher for the smooth case: it represents about $+35 \%$ and $+10 \%$ of the velocity in the constant-velocity region for the smooth and the rough case respectively.

A side view of the velocity bulge phenomenon for test case W0-Q15 is illustrated in Fig. 4b. Two lateral velocity profiles in the wake of a cylinder $(40 \mathrm{~mm}$ downstream of the cylinder centre) are plotted at two different heights: $z / D=1.9$ (where the maximum velocity bulge is observed) and at $z / D=4.1$ (corresponding to the constant-velocity region). The velocity bulge is clearly visible in the cylinder alignment $(y=260 \mathrm{~mm})$ and is associated with a weaker wake at $z / D=1.9$ compared with the wake in the constant-velocity region. On the other hand, in the free stream region between two longitudinal rows of cylinders $(280<y<320 \mathrm{~mm})$, the velocity values at $z / D=1.9$ and $z / D=4.1$ are very similar (despite measurement scatter), indicating that the velocity bulge vanishes in this region.

The velocity bulge phenomenon was first reported in the literature by Liu et al. [14] and was reproduced in LES simulations conducted by Stoesser et al. [26]. In common with our observations, Liu et al. [14] found the velocity bulge to be clearly pronounced in line with a cylinder row and to disappear in the free stream region between two longitudinal rows of cylinders. However, unlike the present study, they measured the maximum bulge closer to the bed at $z / D \approx 0.5$. Liu et al. [14] explain the velocity bulge by the horseshoe vortex at the foot of the cylinder; the legs of this vortex would transport fast fluid from the free stream region to the wake region. However, for a high bed roughness with a characteristic length of the same order of magnitude as the cylinder diameter, we may ponder whether a horseshoe vortex can in fact develop and whether its formation is not hindered by bed-induced turbulent motion.

Figure 5 illustrates the ADV-time series of lateral velocity for test case W0-Q15 at distances of $4 \mathrm{~cm}$ upstream of a cylinder (Point C, Fig. 5a) and of $4 \mathrm{~cm}$ downstream of a cylinder (Point A, Fig. 5b) as well as at four different elevations. Lateral velocity is a good indicator of the presence of periodic coherent structures associated with the von Kármán vortex street. The bed is smooth so the ADV probe measurements in test case W0-Q15 are of good quality even close to the bed. Downstream of the cylinder, clear periodic oscillations of almost constant amplitude can be observed in the constant-velocity region $(z / D=4.1$ and 10.1 in Fig. 5b). These periodic oscillations are dampened, when moving downstream, and become less regular, but they are still visible $4 \mathrm{~cm}$ upstream of the next cylinder $(z / D=4.1$ and 10.1 in Fig. 5a). These oscillations are less and less coherent, when moving downwards, until they almost vanish close to the bed $(z / D=1.0)$, where random fluctuations of bed-induced turbulence can be observed $(z / D=1.0$ in Figs. 5a and $5 b)$.

This analysis infers that the von Kármán vortex street is well developed in the constantvelocity region and is associated with large, high energy vortices, whereas in the boundary layer, the vortex street is unstructured probably by bed-induced turbulence. The drag and energy loss in the cylinder wake are reduced due to the absence of large vortices, which would explain the velocity bulge.

Bed-induced turbulence disorganizes vortex shedding but also causes additional energy loss. This additional energy loss is smaller for a smooth bed than for a rough bed; this fact could explain the higher velocity bulge for a smooth bed (W0-Q15) than for a rough bed (W-Q15).

\subsubsection{Free surface oscillations}

Flows through cylinder arrays are associated with periodic free surface oscillations as observed by previous authors $[30,7,29]$. This physical process has been termed seiching or 
(a)

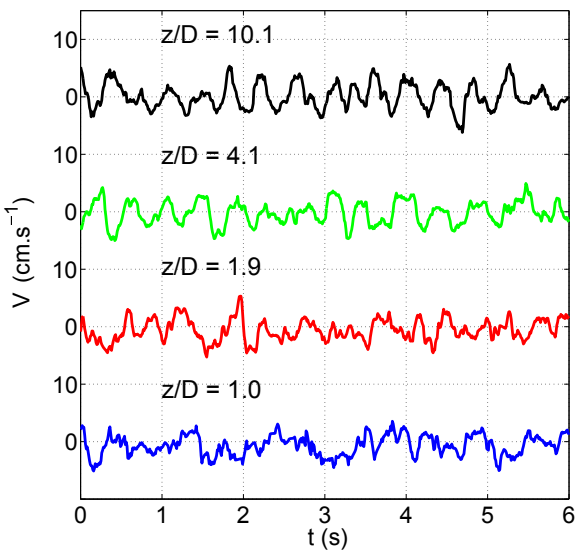

(b)

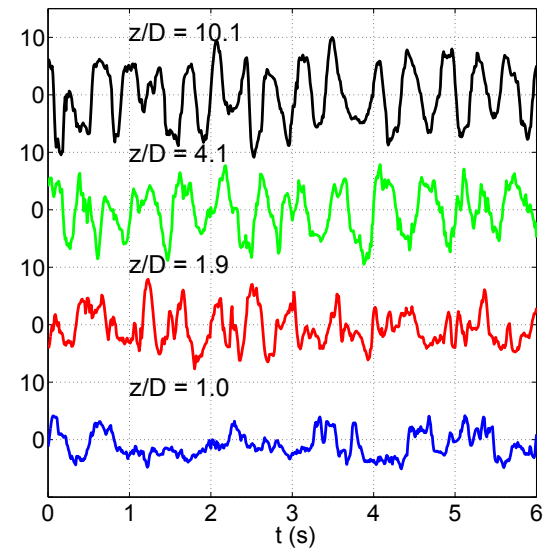

Fig. 5 Time series of lateral velocity for test case W0-Q15: (a) upstream of a cylinder at Point C and (b) downstream of a cylinder at Point A, and at four different heights. The vertical axis origin is shifted for each elevation.

seiche in analogy with the phenomenon occurring in harbours and lakes, although the mechanism generating such oscillations through cylinder arrays is significantly different. In the latter case, seiching results from lift forces induced by the cylinders. Lift forces induced by the different cylinders enter in phase to produce regular transverse standing waves [30]. Seiching in cylinder arrays has been observed for both staggered and in-line cylinder arrangements [11] as well as for randomly distributed cylinders [24].

Zima and Ackermann [30] have provided a physical explanation of the seiche phenomenon: when the vortex shedding frequency $f_{V}$ of each cylinder is close to the natural frequency of the transverse waves in the channel $f_{T W}$, then the energy of these transverse waves is amplified. Resonance occurs between the two oscillations and both frequencies become equal (lock-in process). The natural frequency of the transverse waves can be calculated using the equation derived by Zima and Ackermann [30]:

$$
f_{T W}=\left(\frac{g n_{0}}{4 \pi B} \tanh \frac{n_{0} \pi H}{B}\right)^{1 / 2}
$$

where $n_{0}$ is the oscillation mode.

Zima and Ackermann [30] have derived a formula for predicting the maximum seiching amplitude and Defina and Pradella [7] have rewritten and extended their formula for any oscillation mode $n_{0}$ :

$$
\frac{A_{\text {Zima }}}{H}=2.255 \frac{D}{B} N D^{2} n_{0} \frac{1}{S t^{2}}
$$

where $S t$ is the Strouhal number, the normalised form of the vortex-shedding frequency: $S t=f_{V} D / U_{Q}$.

We also observed free surface oscillations during our experiments. Table 2 summarizes these observations: seiching was observed for the two higher flow rates (W-Q15 and WQ21) but not for the lowest flow rate (W-Q7). Random fluctuations of the free surface only were observed in the latter case. 
Table 2 Seiche parameters for the uniform flow cases through the cylinder array: maximum fluctuation amplitude of water depth $A$ along an antinode line, standard deviation $\sigma_{H m a x}$ along an antinode line at the longitudinal position where it is maximum, seiche amplitude $A_{\text {Zima }}$ derived from Eq. 8, cylinder Reynolds number $\operatorname{Re}_{D}=U_{Q} D / v$, peak frequency of water depth spectra $f_{H}$, peak frequency of velocity spectra $f_{V}$, most appropriate oscillation mode $n_{0}$, natural frequency of transverse waves $f_{T W}$, Strouhal number $S t=f_{V} D / U_{Q}$; 'N.M.' = 'not measured'.

\begin{tabular}{cccccccccccc}
\hline Test case & Seiche & $\begin{array}{c}A \\
(\mathrm{~mm})\end{array}$ & $A / H$ & $\begin{array}{c}\sigma_{\operatorname{Hmax}} \\
(\mathrm{mm})\end{array}$ & $\begin{array}{c}A_{\text {Zima }} \\
(\mathrm{mm})\end{array}$ & $R e_{D}$ & $\begin{array}{c}f_{H} \\
(\mathrm{~Hz})\end{array}$ & $\begin{array}{c}f_{V} \\
(\mathrm{~Hz})\end{array}$ & $n_{0}$ & $\begin{array}{c}f_{T W} \\
(\mathrm{~Hz})\end{array}$ & $S t$ \\
\hline W-Q7 & no & 0.2 & $<0.01$ & 0.2 & 2.4 & 1273 & - & 2.16 & 7 & 2.14 & 0.17 \\
W-Q15 & yes & 10 & 0.09 & 2.5 & 5.0 & 1327 & 2.08 & 2.08 & 6 & 2.13 & 0.16 \\
W-Q21 & yes & 7 & 0.05 & 1.9 & 7.3 & 1382 & 2.14 & N.M. & 6 & 2.16 & 0.15 \\
\hline
\end{tabular}

Seiche frequency $f_{H}$ was evaluated using the water depth fluctuation spectra, in which a clear peak can be distinguished (not shown). Seiche frequency $f_{H}$ is almost the same in flows in which seiching occurs (W-Q15 and W-Q21), and $f_{H} \approx 2.1 \mathrm{~Hz}$. The vortex shedding frequency can be evaluated using the peak frequency $f_{V}$ in the velocity spectrum (not shown). This peak frequency is about $f_{V} \approx 2.1 \mathrm{~Hz}$ for W-Q7 and W-Q15. It was not measured for W-Q21, but it can be assumed to be the same because, according to Section 3.2.2, the constant-velocity region is identical in test cases W-Q15 and W-Q21.

Table 2 reports the natural frequency of transverse waves $f_{T W}$ using oscillation mode $n_{0}$, for which $f_{T W}$ (calculated using Eq. 7) is closest to vortex shedding frequency $f_{V}$. This oscillation mode is a priori the mode most appropriated for seiching. The most appropriated mode appears to be $n_{0}=6$ for W-Q15 and W-Q21 (experimentally confirmed, see below) and $n_{0}=7$ for W-Q7. For these three cases, the corresponding natural transverse wave frequency $f_{T W}$ is very close to the vortex shedding frequency $f_{V}$ and to the seiche frequency $f_{H}$. This confirms the hypothesis of Zima and Ackermann [30] of a lock-in process between natural transverse waves and vortex shedding. However, this cannot explain why the seiche does not occur for the lowest flow rate W-Q7. In common with the two other cases, frequencies $f_{T W}$ and $f_{V}$ are very similar for W-Q7 and conditions are favourable to frequency lock-in.

Absence of seiching for the lower flow rate W-Q7 could be explained by the strong three-dimensional nature of the flow in this case. Figure 4 shows that for W-Q7, the constantvelocity region, in which the von Kármán vortex street is well developed, occupies a very small part of the water column. Below $z=40 \mathrm{~mm}$, the von Kármán vortex street is disorganised due to near-bed turbulence (see Fig. 5 in Section 3.2.2). The lift forces, which are the seiche driving forces, are therefore smaller and less periodic than in flow cases with a large constant-velocity region. This means that the water depth/boundary layer height ratio $(H / \delta)$ is an important parameter for the seiche. No seiche can occur, if this ratio is too close to 1 . For example, there is a seiche for W-Q15, for which $H / \delta=2.05$, but there is no seiche for W-Q7, for which $H / \delta=1.38$.

The seiching in test case W-Q15 was investigated in more detail. The time required to reach a steady state was found to be very long (several hours) as shown in Fig. 6. Twenty minutes after starting the experiment $\left(1.810^{4} \mathrm{D} / U_{\text {drag }}\right)$, the oscillations have a quite constant period but the amplitude fluctuates with time. After five hours $\left(2.610^{5} \mathrm{D} / U_{\text {drag }}\right)$, the amplitude has increased and remains constant. This observation allows us to deduce that bringing all cylinder wakes into phase is a very slow process.

Figure 7 shows two lateral profiles of the flow depth standard deviation, located downstream from an even row (Position 1 in Fig. 1b) and an odd row (Position 2), respectively, 


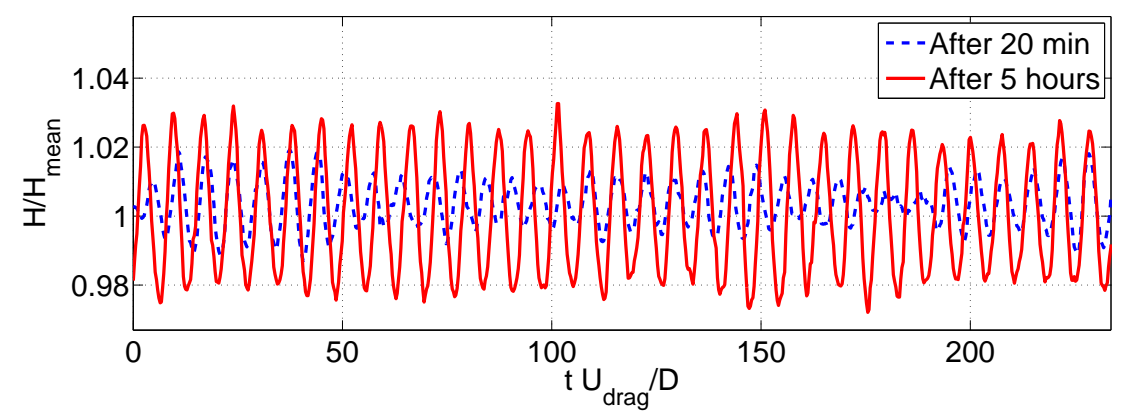

Fig. 6 Time series of normalised flow depth $H / H_{\text {mean }}$ (where $H_{\text {mean }}$ is the time-averaged flow depth) for test case W-Q15, 20 minutes $\left(1.810^{4} \mathrm{D} / \mathrm{U}_{\text {drag }}\right)$ and five hours $\left(2.610^{5} \mathrm{D} / \mathrm{U}_{\text {drag }}\right)$ after starting experiment. Measuring position: $x=-2 \mathrm{~m}, y=450 \mathrm{~mm}$.

and after seiching convergence. The standard deviation profile is the same for the two rows, indicating that the seiche is a purely transversal standing wave, the longitudinal heterogeneity of the flow within the cylinder array having no incidence.

Antinodes are the locations with maximum standard deviation. From Fig. 7 the distance between two antinodes can be evaluated at $165 \mathrm{~mm}$, corresponding to an estimated wavelength of $330 \mathrm{~mm}$. As antinodes also are the locations where the transverse flow rate is zero, two antinodes are located at each side-wall of the flume [20]. Since the channel width is $B=1000 \mathrm{~mm}$, it can be concluded that the mode of oscillation is $n_{0}=6$, which indeed corresponds to the most appropriate oscillation mode calculated above, and there is exactly three wavelengths across the channel, as sketched in Fig. $1 \mathrm{~b}$.

Table 2 reports the maximum seiche amplitude $A$, i.e. the amplitude at an antinode and at mid-length of the cylinder array (see Section 4.3 for the longitudinal variation in seiche amplitude) as well as the seiche amplitude $A_{\text {Zima }}$ predicted using Eq. 8 for the most appropriated mode $n_{0}$. The Zima and Ackermann [30] equation provides an excellent estimation of seiching amplitude for W-Q21, but underestimates by a factor 2 the measured seiching amplitudes for W-Q15. It does not predict that the W-Q15 seiche is larger than the W-Q21 seiche nor that no seiching occurs in test case W-Q7.

\section{Longitudinal roughness transitions}

In this section, we investigate non-uniform flows associated with a longitudinal transition from bed friction to emergent cylinder drag and vice versa. We first analyse the longitudinal 


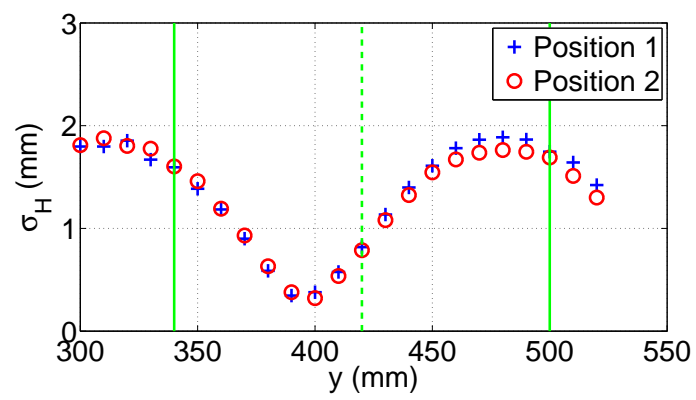

Fig. 7 Lateral variation in standard deviation of flow depth for test case W-Q15 downstream of an even and an odd row (Position 1 and 2, respectively, see Fig. 1b). Dashed and solid lines represent the lateral positions of the cylinders for an even and an odd row, respectively.

variation in water depth, mean velocity and turbulence. The effect of flow non-uniformity on the seiche phenomenon is subsequently considered.

\subsection{Water depth}

Figure 8 shows the longitudinal variations in water depth for the six test cases involving a roughness transition (see Table 1). The water depth varies only upstream of the transition. Downstream, the uniform water depth related to the downstream roughness is immediately reached (compare with uniform water depth in Table 1).

In the following discussion, a simple 1D-momentum equation is introduced to calculate the water depth in a non-uniform flow subjected to bed friction and emergent cylinder drag. Non-uniform open channel flow over a conventional bed roughness is described by the 1D shallow water equation provided by Graf and Altinakar [10], for example. To account for the drag forces exerted by the cylinder array, this equation can be modified (see Dupuis et al. [8] for more detail) into:

$$
\frac{\partial H}{\partial x}\left(1-\frac{Q^{2}}{g B^{2} H^{3}}\right)=S_{0}-\frac{n^{2} Q^{2}}{H^{10 / 3} B^{2}}-\frac{a C_{D} Q^{2}}{2 g H^{2} B^{2}}
$$

in which the drag force term is the third term on the right-hand side of the equation. The second term on the right-hand side is the bed friction term, expressed using the Manning coefficient $n$. The values of $n$ and $C_{D}$ provided by uniform flow cases (see Section 3) are used to solve Eq. 9. These solutions have been plotted as dotted lines in Fig. 8. Computation starts from the most downstream measuring point. This value does not exactly match the uniform water depth, so a slight water depth variation can be sometimes observed in the downstream reach. Moreover, discrepancies between the model and the measurements may be related to the Eq. 3 insufficiency for modelling bed shear stress with an emergent cylinder array (second term on the right-hand side of Eq. 9). Very satisfactory results are nevertheless obtained: the model reproduces the measuring points with a mean relative error of $\varepsilon=0.9 \%$ and a maximum relative error of $\varepsilon_{\max }=3.8 \%$.

The 1D-momentum equation was used to extrapolate the longitudinal profiles of water depth further upstream. The results are shown in Fig. 9 in non-dimensional form: $H_{u p}$ and $H_{d w}$ refer to the uniform water depth associated with the upstream and downstream roughnesses respectively. The distance required to reach equilibrium upstream of the transition 

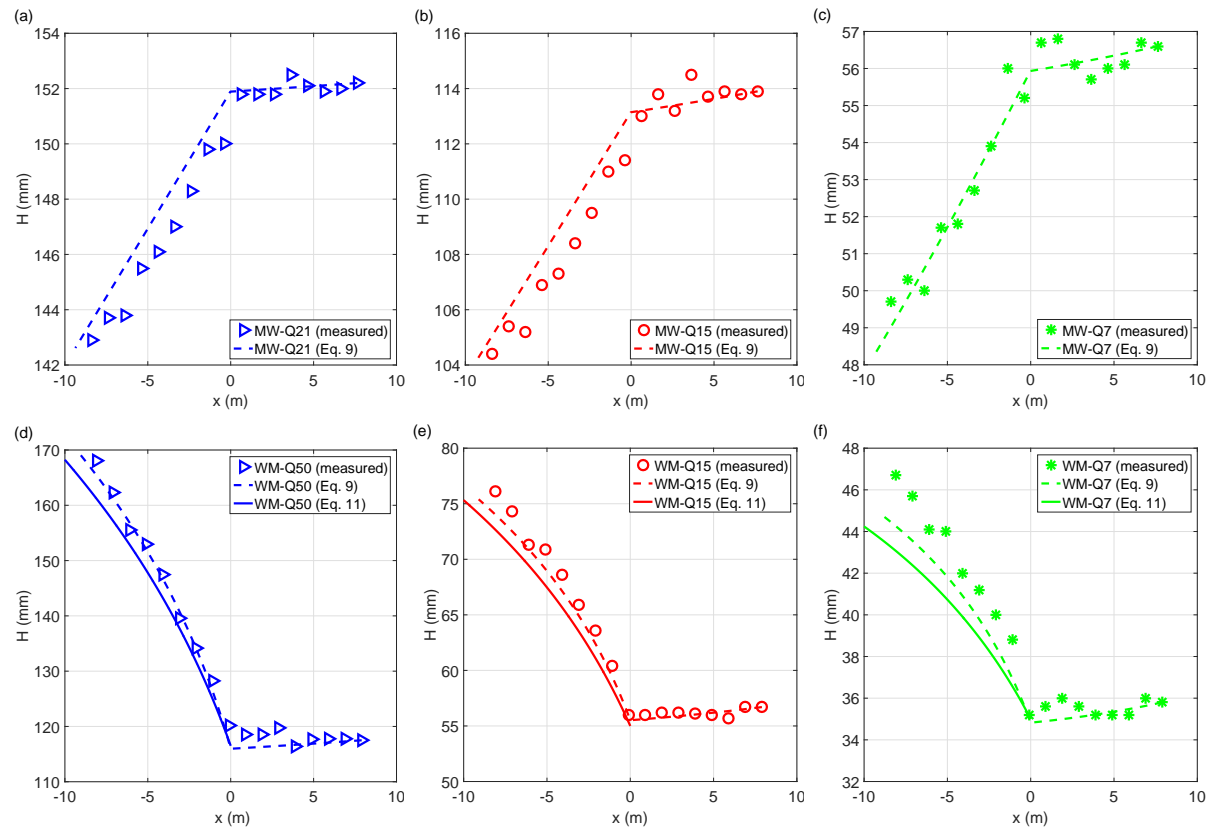

Fig. 8 Longitudinal profiles of water depth for six test cases involving a roughness transition. Symbols are measuring points, dashed lines are numerical solutions of Eq. 9 and solid lines are numerical solutions of Eq. 11.

$L_{u p}$ is defined as the distance, at which the water surface slope is $|d H / d x|=10^{-6}$. For the six flow cases, $L_{u p} / H_{u p}=2580 \pm 15 \%$ and thus $L_{u p}$ approximately scales with $H_{u p}$. This implies that, for a given flow rate, a roughness step change with downstream increasing roughness will reach equilibrium over a shorter distance than a roughness step change with downstream decreasing roughness.

When bed friction is negligible compared to drag forces $(R<0.1)$, the bed friction terms in Eqs. 2 and 9 can be assumed to be zero. Combining these equations then gives:

$$
\frac{\partial \bar{H}}{\partial \bar{x}}\left(1-F r^{2}\right)=S_{0}\left(1-\frac{1}{\bar{H}^{2}}\right)
$$

with $\bar{H}=H / H_{u p}$ and $\bar{x}=x / H_{u p}$. Considering that $\left(1-F r^{2}\right) \approx c s t \approx 1$, which is a good approximation for flows in cylinder arrays (see Table 1), Eq. 10 can be solved analytically (but implicitly):

$$
\frac{H-H_{d w}}{H_{u p}}-\operatorname{atanh} \frac{H}{H_{u p}}+\operatorname{atanh} \frac{H_{d w}}{H_{u p}}=S_{0} \frac{x}{H_{u p}} .
$$

Equation 11 has been plotted for the three wood-to-meadow cases illustrated in Figs. 8d, $8 \mathrm{e}$ and $8 \mathrm{f}$ as solid lines. Since the analytical equation neglects bed friction, it has been applied only to the wood area $(x<0)$. The analytical solution reproduces the measurements for test cases WM-Q50 and WM-Q15 with mean relative errors of $\varepsilon=2.4 \%$ and $\varepsilon=3.6 \%$, respectively. For test case WM-Q7, the errors are larger $(\varepsilon=5.5 \%)$ since bed roughness has a greater influence $(R \approx 0.4)$. 


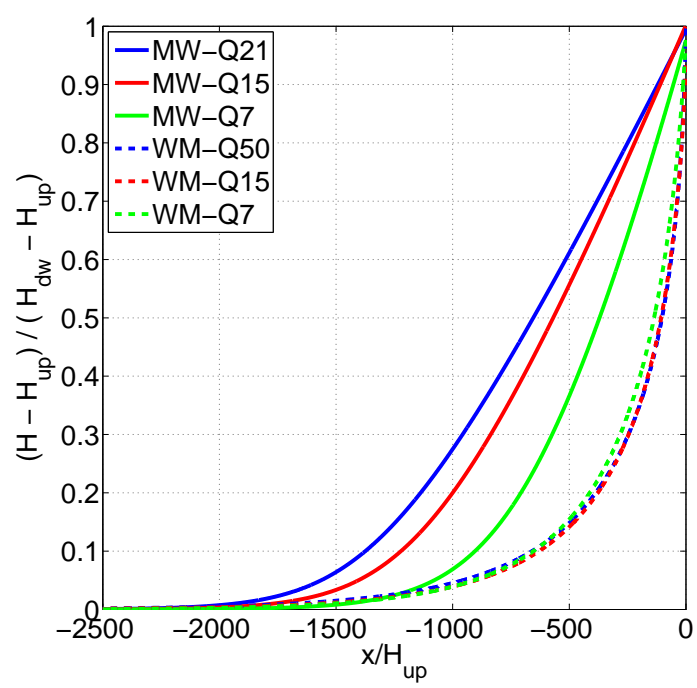

Fig. 9 Extrapolated backwater curves for the six roughness transitions studied (upstream of roughness transition).

\subsection{Mean velocity and turbulence}

Figures 10a and 10b show vertical profiles of mean longitudinal velocity for test case MWQ15 upstream and downstream of the roughness transition. Measurement was performed at Point B within the cylinder array, i.e. in the free stream region between two longitudinal rows of cylinders. Velocity $U$ is normalised by the local bulk velocity $U_{Q}$ and elevation $z$ by the local water depth $H$. The collapse of the normalised velocity profiles upstream of the transition (Fig. 10a) indicates flow self-similarity. No shape deviation was observed even very close to the roughness step change.

Figure 10a also shows the uniform velocity profile over the meadow (taken from test case M-Q50 and measured with PIV). The normalised profiles of test case MW-Q15 collapse with the normalised uniform flow profile. Depth-averaged flow MW-Q15 is decelerating (water depth increasing in the upstream reach when going downstream). Kironoto and Graf [12] have shown that there is a deviation from the uniform normalised profile, when flows are sufficiently accelerated or decelerated. In this case, flow deceleration upstream of the transition is not large enough for deviation from the uniform distribution.

Figure 10 shows that longitudinal changes in the mean velocity profile are only observed downstream of the transition (Fig. 10b), where the logarithmic profile changes gradually into the characteristic constant velocity profile of the wood (blue crosses indicate the uniform flow profile).

Figure 11 shows the vertical profiles of longitudinal turbulence intensity at the same $x$-positions as in Fig. 10. The turbulence intensity is normalised by the local shear velocity $U^{*}=\left(g H S_{f}\right)^{1 / 2}$, where $S_{f}$ is the equivalent bed friction slope determined from the Manning equation $S_{f}=n^{2} U_{Q}^{2} / H^{4 / 3}$. In common with the velocity, normalised turbulence intensity is relatively self-similar upstream of the transition (Fig. 11a) and the vertical distribution is close to the uniform one. Downstream of the transition (Fig. 11b), the turbulence intensity profile adapts to the downstream roughness: starting with an exponential form characteristic 
(a)

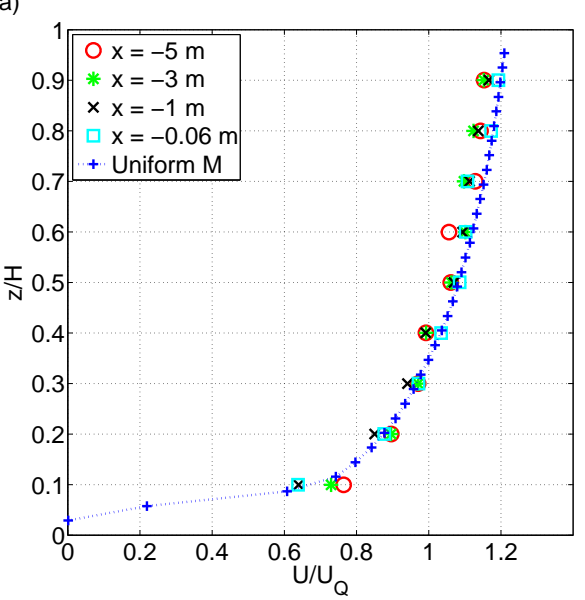

(b)

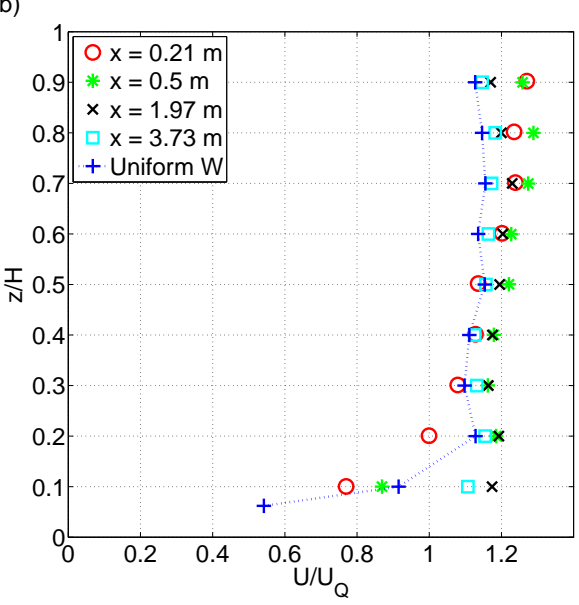

Fig. 10 Vertical profiles of mean longitudinal velocity normalised by local bulk velocity at various distances $x$ from transition: (a) upstream and (b) downstream of the roughness transition for test case MW-Q15. Blue crosses correspond to uniform flows over the meadow (M-Q50 - PIV) for panel a and in the wood (W-Q15) for panel $b$.

(a)

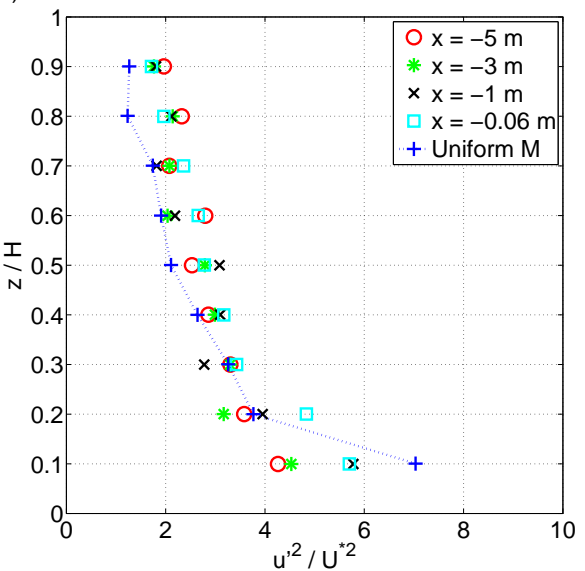

(b)

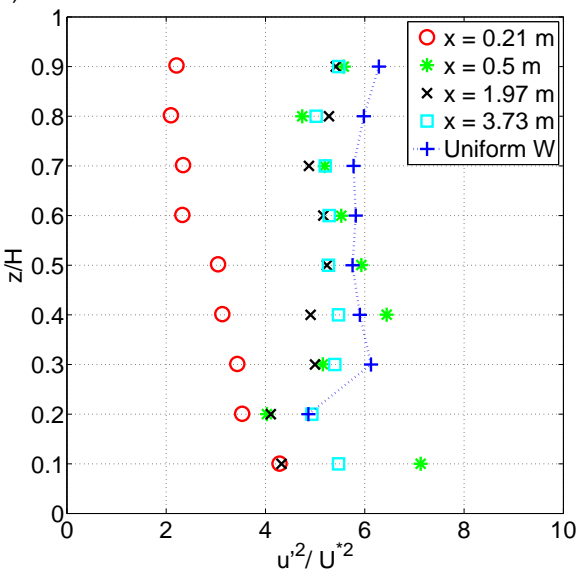

Fig. 11 Vertical profiles of longitudinal turbulence intensity normalised by local shear velocity at various distances $x$ from the transition: (a) upstream and (b) downstream of the roughness transition for test case MW-Q15. Blue crosses correspond to uniform flows over the meadow (M-Q50) for panel a and in the wood (W-Q15) for panel b.

of bed roughness [17], which persists at $x=0.21 \mathrm{~m}$, the turbulence intensity increases and becomes uniformly distributed along the water column.

The above flow dynamics for the meadow-to-wood transition were also observed for the reverse wood-to-meadow transition (not shown here, see Dupuis et al. [8]). The flow is selfsimilar upstream of the transition, the normalised profiles collapse with the uniform flow profile; downstream of the transition, the vertical profile changes gradually into the uniform 
profile of downstream roughness. As observed by Chen and Chiew [4], the vertical profile downstream of the transition is simultaneously transformed across the entire flow depth and not gradually from the wall to the top of the boundary layer, as is the case for air flows.

For the three transition flows investigated with the ADV probe (MW-Q15,WM-Q15 and WM-Q50), the downstream distance $L_{d w}$ from the transition before reaching the uniform flow (for mean velocity and turbulence) can be estimated as $L_{d w} / H_{d w} \approx 35-50$. No effect of flow rate and transition type could be established over this distance, but further experiments with more configurations and with a more precise determination of $L_{d w}$ are required to be able to conclude on this issue.

\subsection{Free surface oscillations}

The seiche phenomenon (see Section 3.2.3) was also observed when there was a roughness transition. The free surface oscillations in meadow-to-wood transition MW-Q15 were studied in detail to examine how flow non-uniformity affects seiching. Defina and Pradella [7] theoretically showed that a longitudinal water depth gradient should reduce seiching.

Figure 12 illustrates the longitudinal variation in flow depth standard deviation, along an antinode line for a uniform flow through a cylinder array and for a meadow-to-wood transition under the same flow rate (test cases W-Q15 and MW-Q15). For the uniform flow case, the seiche longitudinal profile is triangular with a maximum intensity almost at midlength of the cylinder array. For MW-Q15 (cylinder array occupying only second half of flume), the seiche intensity decreases dramatically outside the cylinder array. However, the time series of the free surface elevation show that seiching persists outside the cylinder array. Coherent free surface oscillations with the same frequency were observed as far as the upstream end of the channel, although their amplitudes become very small. Within the cylinder array, the seiche intensity was also triangular with a maximum intensity at midlength of the cylinder array.

Sarkar [24] has also noted an almost triangular shape of the seiche amplitude longitudinal profile. However, Sarkar finds the maximum amplitude to be at one-fifth of the total length of the cylinder array, which is different to our finding that the maximum amplitude is at mid-length. This difference could be attributed to non-uniformity of the flow within the cylinder array in Sarkar's experiments.

The peak intensity of the seiche is lower in roughness transition case MW-Q15 than in uniform flow case W-Q15. This may be due to the shorter length of the cylinder array. Flow non-uniformity in the first metres after the meadow-to-wood step change in test case MW-Q15 (see Section 4.2) may also contribute to lower peak seiche intensity.

Figure 12 indicates that the seiche amplitude is closely dependent on longitudinal position within the array. However, this parameter is never considered in the expressions that can be found in the literature for evaluating seiche amplitude (reviewed by Defina and Pradella [7]). Figure 12 also shows that the length of the cylinder array influences the maximum oscillation amplitude. This differs from the result of Zima and Ackermann [30] who stated that when the cylinder array is longer than a minimum length of the order of six cylinder rows, the wave amplitude is independent on the cylinder array length. These facts could partly explain the wide variation in seiche amplitude measurements found in the literature. The values presented in Table 2 are the maximum seiche amplitudes over the channel length.

The flow accelerates within the cylinder array and the water depth varies in the woodto-meadow transition. This means that the vortex shedding and the natural frequency of the transverse waves vary in the longitudinal direction. If the vortex shedding frequency 


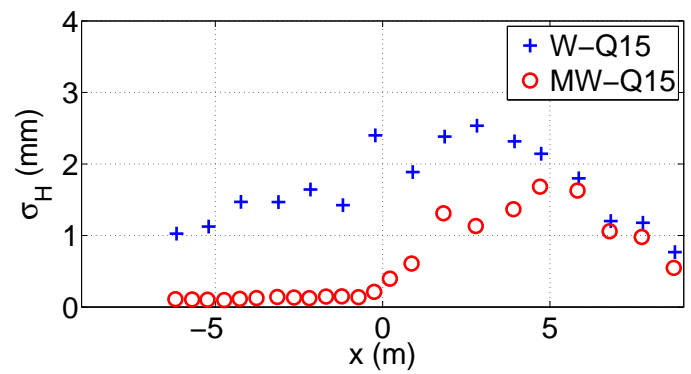

Fig. 12 Longitudinal variation in flow depth standard deviation $\sigma_{H}$ along an antinode line for a uniform flow (W-Q15) and a roughness transition (MW-Q15) under the same flow rate $Q=15 \mathrm{~L} \cdot \mathrm{s}^{-1}$.

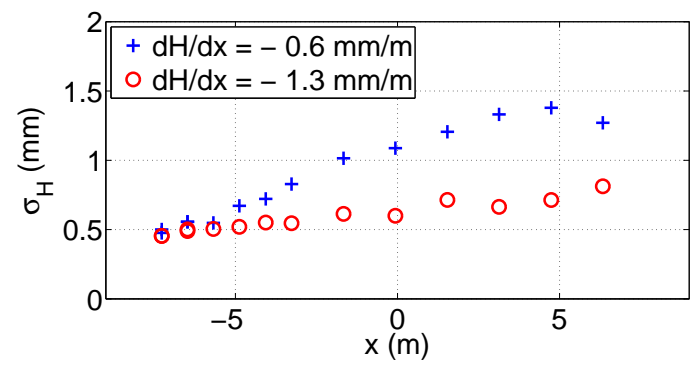

Fig. 13 Longitudinal variation in flow depth standard deviation $\sigma_{H}$ along an antinode line for two accelerated flows with water depth gradients of $d H / d x=-0.6 \mathrm{~mm} \cdot \mathrm{m}^{-1}$ and $d H / d x=-1.3 \mathrm{~mm} \cdot \mathrm{m}^{-1}$ respectively. Flow rate is $Q=15 \mathrm{~L} \cdot \mathrm{s}^{-1}$. Cylinder array is $18 \mathrm{~m}$ long.

varies too much, no resonance can be expected to occur on the array scale. To test this hypothesis (seiching was unfortunately not investigated for the WM cases), we studied two accelerated flows in a cylinder array occupying the whole flume under the same flow rate $Q=15 \mathrm{~L} \cdot \mathrm{s}^{-1}$ : a lower acceleration with a water depth gradient of $d H / d x=-0.6 \mathrm{~mm} \cdot \mathrm{m}^{-1}$ $(H(x=0)=92 \mathrm{~mm})$ and a higher acceleration with a water depth gradient of $d H / d x=$ $-1.3 \mathrm{~mm} \cdot \mathrm{m}^{-1}(H(x=0)=84 \mathrm{~mm})$. The accelerated flows were obtained by lowering the flume downstream weir. Figure 13 illustrates the flow depth standard deviations for these two accelerated flows. The variations in Strouhal frequency, using $f_{S t}=0.2 U_{Q} / D$, for these accelerations are $d f_{S t} / d x=0.021 \mathrm{~Hz} . \mathrm{m}^{-1}$ and $d f_{S t} / d x=0.055 \mathrm{~Hz} . \mathrm{m}^{-1}$ respectively.

Periodic free surface oscillations were observed in the water depth time series. However, the fluctuation amplitudes were lower than those in the uniform flow case (W-Q15): the amplitudes are approximately one half and one quarter of those of the uniform flow case for the lower and higher accelerations respectively. The water depth time series show that the frequency along the flume is constant for both flows. It can be concluded that, although the seiche can still be observed when the flow is non-uniform within the cylinder array, its intensity is greatly reduced. 


\section{Conclusion}

The combined effects of bed friction and cylinder drag were analysed for uniform flows through an array of emergent cylinders installed on a rough bed (dense meadow) or a smooth bed (glass bottom). The water column within a cylinder array could be divided into a constant-velocity region in the upper water column and a boundary layer in the near-bed region. The boundary layer height was found to be independent of the water depth and of the bed surface condition (rough or smooth). Conversely, the shape of the boundary layer was dependent on the bed roughness. A local increase in longitudinal velocity (velocity bulge) was observed near the bed in line with a longitudinal row of cylinders. This velocity bulge may be related to the effect of bed-induced turbulence. The latter disorganizes the von Kármán vortex street, resulting in smaller drag forces and less momentum loss in the cylinder wake. Additional energy loss is due to the bed friction in the near-bed region. Friction is higher for a rough bed than for a smooth bed, so near-bed velocity is lower and velocity bulge is weaker in the case of a rough bed.

Longitudinal roughness transitions between bed friction and emergent cylinder drag, and vice versa, were then investigated. Flow depth and velocity responses to roughness transitions were found to oppose each other. The water depth varied upstream of a roughness transition, while the vertical profiles of mean longitudinal velocity and turbulence remained constant and similar to uniform flow profiles, if the former parameters were normalised by the local section-averaged mean velocity and the local shear velocity respectively. The water depth was constant downstream of the transition, while a vertical redistribution of mass and momentum occurred over a longitudinal distance of $L_{d w} / H_{d w} \approx 35-50$, where $H_{d w}$ is the uniform flow depth of the downstream roughness.

A 1D momentum equation including a volume drag force was used to predict the water depth profile with a mean error of $0.9 \%$. This equation was also used to calculate the distance upstream of the transition to reach equilibrium $L_{u p}$. This distance scaled approximately with the uniform flow depth of the upstream roughness $H_{u p}$ and $L_{u p} / H_{u p} \approx 2580$. The momentum equation was analytically solved for zero bed friction.

Free surface oscillations (seiching) were observed through the cylinder array under uniform and non-uniform flow conditions. Several hours were required to achieve constant amplitude oscillations. The amplitude was found to be dependent on the longitudinal position within the array with a peak value at its mid-length. Furthermore, the seiching decreased with a decreasing array length. The seiche driving forces are the periodic lift forces in the cylinder wake. These were found to be only present in the constant-velocity region, whereas they were prevented in the boundary layer because of the disorganization of the von Kármán vortex street. Therefore, if the water depth/boundary layer height ratio was close to unity, no seiche appeared. Finally, the seiching process was found to decrease with increasing flow non-uniformity. This could be associated with the increasing Strouhal frequency gradient along the cylinder array, which curtails synchronization between cylinder wakes.

Acknowledgements The authors wish to thank Fabien Thollet for his technical support and Sheng Chen for his relevant suggestions. The PhD grant of V. Dupuis was funded by Irstea and by the French National Research Agency (Flowres project, grant No. ANR-14-CE03-0010, http://flowres.irstea.fr/).

\section{References}

1. Antonia, R., Luxton, R.: The response of a turbulent boundary layer to a step change in surface roughness Part 1. Smooth to rough. Journal of Fluid Mechanics 48(04), 721-761 (1971) 
2. Carravetta, A., Della Morte, R.: Response of velocity to a sudden change of bed roughness in sub critical open channel flow. In: River Flow 2004: Proceedings of the Second International Conference on Fluvial Hydraulics, 23-25 June 2004, Napoli, Italy, Two Volme Set, p. 389. CRC Press (2004)

3. Chanson, H., Trevethan, M., Koch, C.: Discussion of "Turbulence measurements with acoustic Doppler velocimeters" by C. M. García, M. I. Cantero, Y. Niño, and M. H. García. Journal of Hydraulic Engineering 133(11), 1283-1286 (2007)

4. Chen, X., Chiew, Y.M.: Response of velocity and turbulence to sudden change of bed roughness in open-channel flow. Journal of Hydraulic Engineering 129(1), 35-43 (2003)

5. Chen, X., Chiew, Y.M.: Closure to "response of velocity and turbulence to sudden change of bed roughness in open-channel flow" by xingwei chen and yee-meng chiew. Journal of Hydraulic Engineering 130(6), 589-590 (2004)

6. Cheng, H., Castro, I.P.: Near-wall flow development after a step change in surface roughness. BoundaryLayer Meteorology 105(3), 411-432 (2002)

7. Defina, A., Pradella, I.: Vortex-induced cross-flow seiching in cylinder arrays. Advances in Water Resources 71, 140-148 (2014)

8. Dupuis, V., Proust, S., Berni, C., Paquier, A., Thollet, F.: Open-channel flow over longitudinal roughness transition from highly-submerged to emergent vegetation. 36th IAHR World Congress, The Hague (2015)

9. Goring, D.G., Nikora, V.I.: Despiking acoustic Doppler velocimeter data. Journal of Hydraulic Engineering 128(1), 117-126 (2002)

10. Graf, W.H., Altinakar, M.S.: Fluvial hydraulics. Wiley (1998)

11. Jafari, A., Ghomeshi, M., Bina, M., Kashefipour, S.M.: Experimental study on ten modes of transverse waves due to vertical cylinders in open channels. Journal of Food, Agriculture \& Environment 8(2), 949-955 (2010)

12. Kironoto, B., Graf, W.H.: Turbulence characteristics in rough uniform open-channel flow. Proceedings of the ICE-Water Maritime and Energy 106(4), 333-344 (1994)

13. Kothyari, U.C., Hayashi, K., Hashimoto, H.: Drag coefficient of unsubmerged rigid vegetation stems in open channel flows. Journal of Hydraulic Research 47(6), 691-699 (2009)

14. Liu, D., Diplas, P., Fairbanks, J., Hodges, C.: An experimental study of flow through rigid vegetation. Journal of Geophysical Research: Earth Surface (2003-2012) 113(F4) (2008)

15. Martino, R., Paterson, A., Piva, M.: Double-average mean flow and local turbulence intensity profiles from PIV measurements for an open channel flow with rigid vegetation. Environmental Fluid Mechanics 12(1), 45-62 (2012)

16. Nepf, H.: Drag, turbulence, and diffusion in flow through emergent vegetation. Water Resources Research 35(2), 479-489 (1999)

17. Nezu, I., Rodi, W.: Open-channel flow measurements with a laser Doppler anemometer. Journal of Hydraulic Engineering 112(5), 335-355 (1986)

18. Pendergrass, W., Arya, S.: Dispersion in neutral boundary layer over a step change in surface roughness-I. Mean flow and turbulence structure. Atmospheric Environment (1967) 18(7), 1267-1279 (1984)

19. Poggi, D., Porporato, A., Ridolfi, L., Albertson, J., Katul, G.: The effect of vegetation density on canopy sub-layer turbulence. Boundary-Layer Meteorology 111(3), 565-587 (2004)

20. Rabinovich, A.B.: Seiches and harbor oscillations. Handbook of Coastal and Ocean Engineering pp. 193-236 (2009)

21. Raupach, M., Antonia, R., Rajagopalan, S.: Rough-wall turbulent boundary layers. Applied Mechanics Reviews 44(1), 1-25 (1991)

22. Robert, A., Roy, A.G., de Serres, B.: Changes in velocity profiles at roughness transitions in coarse grained channels. Sedimentology 39(5), 725-735 (1992)

23. Rominger, J.T., Nepf, H.M.: Flow adjustment and interior flow associated with a rectangular porous obstruction. Journal of Fluid Mechanics 680, 636-659 (2011)

24. Sarkar, A.: Vortex-excited transverse surface waves in an array of randomly placed circular cylinders. Journal of Hydraulic Engineering 138(7), 610-618 (2012)

25. Siuru, W., Logan, E.: Response of a turbulent pipe flow to a change in roughness. Journal of Fluids Engineering 99(3), 548-553 (1977)

26. Stoesser, T., Kim, S., Diplas, P.: Turbulent flow through idealized emergent vegetation. Journal of Hydraulic Engineering (2010)

27. Terrier, B.: Flow characteristics in straight compound channels with vegetation along the main channel. Ph.D. thesis, Loughborough University (2010)

28. Yen, B.C.: Open channel flow resistance. Journal of Hydraulic Engineering 128(1), 20-39 (2002)

29. Zhao, K., Cheng, N.S., Huang, Z.: Experimental study of free-surface fluctuations in open-channel flow in the presence of periodic cylinder arrays. Journal of Hydraulic Research 52(4), 465-475 (2014) 
30. Zima, L., Ackermann, N.L.: Wave generation in open channels by vortex shedding from channel obstructions. Journal of Hydraulic Engineering 128(6), 596-603 (2002)

31. Zong, L., Nepf, H.: Flow and deposition in and around a finite patch of vegetation. Geomorphology 116(3), 363-372 (2010) 